\title{
Commentary: "Dynamin 1 isoform roles in a mouse model of severe childhood epileptic encephalopathy"
}

\author{
Narayan Subramanian ${ }^{1}$, Wayne N. Frankel ${ }^{1,2 *}$
}

'Institute for Genomic Medicine, Columbia University Medical Center, New York, NY 10032

${ }^{2}$ Department of Genetics \& Development, Columbia University Medical Center, New York, NY 10032

\section{Article Info}

Article Notes

Received: August 29, 2016

Accepted: September 23, 2016

\section{*Correspondence:}

Dr. Wayne N. Frankel,

Columbia University Medical Center, New York, NY 10032

E-mail:wf2218@cumc.columbia.edu

(c) 2016 Wayne N. Frankel. This article is distributed under the terms of the Creative Commons Attribution 4.0 International License.
Epilepsy is a neurological disorder that is characterized by periodic seizures in affected individuals. The cellular mechanisms during a seizure entail synchronous but abnormal firing of neural networks in the higher centers of the brain. Very early studies on cellular mechanisms of epilepsy especially focused on inducing seizures in animal models by blocking synaptic activity using drugs that either block inhibitory neural networks ${ }^{1}$ or activate voltage gated channels ${ }^{2}$ to increase excitation. Many of a growing number of genetically solved epilepsies and their respective animal models have also shown or presume that defective inhibition (leading to excessive excitation) is at the heart of the pathophysiology. In a recent issue of Neurobiology of Disease, Asinof and colleagues stir the pot further with new data in a mouse model of a relatively new and severe form of human epilepsy - DNM1 (dynamin 1) epileptic encephalopathy (EE).

The GTPase dynamin is a crucial protein essential for endocytosis. Of the three human dynamin genes, DNM1 and DNM3 encode the only neuronal GTPases that participate in clathrin mediated synaptic vesicle endocytosis at the pre-synaptic and post-synaptic membrane respectively ${ }^{3}$, while DNM2 is expressed ubiquitously ${ }^{4}$. Although cellular and molecular characterization of dynamin in endocytosis is well documented, its role in neurological diseases has been less ventured. The first direct hint that DNM1 is involved in epilepsy came from a spontaneous mutation (A408T, also known as "fitful") in mouse Dnm1 leading to an EE-like disease when homozygous, and idiopathic epilepsy when heterozygous 5 . More recent human exome sequencing indeed confirmed DNM1 as a bona-fide EE gene with additional cases coming to light ${ }^{6,7}$. The neuropathological and seizure phenotypes exhibited by homozygous fitful mice closely resemble symptoms exhibited by DNM1 individuals with heterozygous de novo variants ${ }^{8}$. The gene dosage difference between species may be explained by the fact that the fitful mutation resides in an alternate exon representing only one of the two major Dnm1 isoforms (we refer readers to Figure 1a of Asinof et al. ${ }^{9}$ illustrating the location of variants within dynamin-1 functional domains and the respective alternate exon pair). In fact, it is probably because of this bit of good fortune that fitful remains the only animal model of DNM1 epileptic encephalopathy - as it seems that a delicate balance and interplay between isoforms is the difference between predisposition and full disease. Although these and other Dnm1 isoforms were previously known, the illustration of functional differences between two particular splice variants is the major take-home message of Asinof et al. ${ }^{9}$. 
Each pathogenic human $D N M 1$ variant described to date is in one of two portions of the protein - residing in either the self-explanatory GTPase domain, or in the "middle" domain $^{10}$, required for oligomerization and self-assembly ${ }^{11}$ of constricting spring like structures, that spiral around the neck of invaginated, clathrin-coated, recycling presynaptic vesicles $^{12}$. Fitful itself resides in only Dnm1a, one of two mutually-exclusive isoforms (its complement is Dnm1b) that differ by an alternate exon in the region of the gene encoding the middle domain. The molecular pathology described in the original fitful publication gibes with impaired middle domain function - mutant Dnm1a cannot self-assemble and actually blocks endocytosis in COS7 cells 5 . Consequently, neurons in fitful homozygous cortical slices show decreased inhibitory neurotransmission with repeated stimulation, presumably due to impaired recycling and thus depletion of GABA-containing synaptic vesicles $^{5}$. A subsequent study used conditional mutant genotypes to confirm that the lethal seizure phenotype of homozygous fitful mice is indeed cell-autonomous to inhibitory neurons ${ }^{13}$.

Now Asinof et al. ${ }^{9}$ provide an interesting link between the molecular and physiological mechanism, while highlighting functional differences between the isoforms. Dnm1a and Dnm1b isoforms are known to have overlapping but different developmental expression. Dnm1b is highest during early embryonic development and it declines towards early adulthood; Dnm1a shows a complementary pattern. This must be why viability of fitful mice (mutant Dnm1a) is rescued by Dnm1b until weaning age, which further implies that Dnm1a is crucial for synapse maturation and Dnm1b cannot fully compensate for the absence of Dnm1a. Subcellular localization of Dnm1a and Dnm1b suggests that both isoforms specifically bind to clathrin, although Dnm1b uniquely localizes near the perinuclear region ${ }^{9}-$ a further hint that the two are functionally distinct.

A significant molecular finding of Asinof et al. ${ }^{9}$ is the apparently selective interaction of amphiphysin with Dnm1a. Recruitment of dynamin to the synaptic membrane was first shown to be regulated by amphiphysin, a SH3 domain containing protein that binds with high affinity with the proline-rich domain of $D N M 1^{14,15}$. This interaction, further coupled with the BAR complex of clathrin mediated endocytosis, is necessary for tubulation, pinching off the vesicle and linking membranes to the actin cytoskeleton ${ }^{16}$. In Dnm1 isoform protein interactions, absence of Dnm1a showed a consistent decrease in binding with amphiphysin, even in the presence of Dnm1b. Also in comparison to the expression of Dnm1a or Dnm1b in COS7 cells, subcellular localization of Dnm1 $1 \mathrm{a}^{\mathrm{Fttl}}$ in COS7 cells revealed a tubular appearance ${ }^{5}$. This is consistent with earlier electron microscopy and in vitro studies of conditional Dnm1 knockout mice that showed that absence of Dnm1 results in endocytic tubulated clathrin-coated pits, and these sites are enriched in BAR protein complex ${ }^{17}$. Thus, Dnm1a is important isoform for binding with the amphiphysin in the BAR complex and for efficient formation of constricted rings around the clathrin coated synaptic vesicles ${ }^{18}$.

The furtherupregulation ofDnm1bin onlyfitful homozygous ${ }^{5}$ and compound heterozygous mice, Dnm $1^{\text {null/Ftt }, 9}$ appears to highlight cellular efforts for Dnm1b to compensate and restore Dnm1 function. Nevertheless, while overall Dnm1 levels are maintained, absence of wildtype Dnm1a in fitful homozygotes leads to lethal seizures. However, a final puzzling observation is the lack of lethal seizures in mice that fully lack Dnm1a; again, Dnm1 $\mathrm{a}^{\text {Ftl }}$ must be present for the seizure disorder. This may well mean that Dnm1b also has a compensatory ability to rescue Dnm1a function in maturing neurons, but that such a role is eventually overcome by the dominant-negative effect of Dnm $1 \mathrm{a}^{\mathrm{Ftfl}}$. Thus, while Dnm1a isoform binding to amphiphysin highlights the importance of Dnm1a interactions with known molecular players in presynaptic endocytic complexes, one way or another Dnm1b may help neurons to find other ways to recycle synaptic vesicles presynaptically, perhaps even via lower affinity interactions with some of the same players.

Although there is still only a modest number pathogenic DNM1 variants known, a trend is emerging whereby those in the GTPase domain appear to confer milder and later onset of symptoms than middle domain; G359A, for example, confers high voltage and slow spike wave discharges as early as 2 months of age in comparison to late onset of seizures conferred by GTPase domain variants ${ }^{7}$; G397D conferred seizures from 6 months and symptoms earlier (personal comm. from the individual's parent) and H396D arose in a study of severe undiagnosed developmental disorders ${ }^{19} \quad$ (http://databases.lovd.nl/ shared/variants/0000079504\#00006594). This trend, if it continues makes sense as variant isoforms that remain as monomers (i.e. middle domain assembly defects) may be harder to rescue. Thus it may be worth studying both GTPase and middle domain mutations in mice to model each type. Nevertheless, like most other EE cases, all individuals with pathogenic DNM1 variants have a poor prognosis and no known drug therapies. The demonstration by Asinof et al. ${ }^{9}$ for a critical role of Dnm1a and interactions with amphiphysin suggest that any successful therapy would either have to target Dnm1a specifically (eliminating mutant Dnm1a, or boost the expression of the wildtype copy in a heterozygote), or enable or stabilize interactions with molecules such as amphiphysin.

\section{References}

1. Matsumoto H, Ajmonemarsan C. Cellular Mechanisms in Experimental Epileptic Seizures. Science. 1964; 144(3615): 193-4. 
2. Walther $\mathrm{H}$, et al. Epileptiform activity in combined slices of the hippocampus, subiculum and entorhinal cortex during perfusion with low magnesium medium. Neurosci Lett. 1986; 69(2): 156-61.

3. Romeu A, Arola L. Classical dynamin DNM1 and DNM3 genes attain maximum expression in the normal human central nervous system. BMC Res Notes. 2014; 7: 188.

4. Cook TA, Urrutia R, McNiven MA. Identification of dynamin 2, an isoform ubiquitously expressed in rat tissues. Proc Natl Acad Sci U S A. 1994; 91(2): 644-8.

5. Boumil RM, et al. A missense mutation in a highly conserved alternate exon of dynamin-1 causes epilepsy in fitful mice. PLoS Genet. 2010; $6(8)$

6. Euro, E.-R.E.S.C., P. Epilepsy Phenome/Genome, and K.C. Epi, De novo mutations in synaptic transmission genes including DNM1 cause epileptic encephalopathies. Am J Hum Genet. 2014; 95(4): 360-70.

7. Nakashima M, et al. De novo DNM1 mutations in two cases of epileptic encephalopathy. Epilepsia. 2016; 57(1): e18-23.

8. Epi KC, et al. De novo mutations in epileptic encephalopathies. Nature. 2013; 501(7466): 217-21.

9. Asinof S, et al. Dynamin 1 isoform roles in a mouse model of severe childhood epileptic encephalopathy. Neurobiol Dis. 2016; 95: 1-11.

10. Dhindsa RS, et al. Epileptic encephalopathy-causing mutations in DNM1 impair synaptic vesicle endocytosis. Neurol Genet. 2015; 1(1): e4.

11. Ramachandran R, et al. The dynamin middle domain is critical for tetramerization and higher-order self-assembly. EMBO J. 2007; 26(2): $559-66$.

12. Sever S, Damke H, Schmid SL. Garrotes, springs, ratchets, and whips: putting dynamin models to the test. Traffic. 2000; 1(5): 385-92.

13. Asinof SK, et al. Independent Neuronal Origin of Seizures and Behavioral Comorbidities in an Animal Model of a Severe Childhood Genetic Epileptic Encephalopathy. PLoS Genet. 2015; 11(6): e1005347.

14. Grabs D, et al. The SH3 domain of amphiphysin binds the proline-rich domain of dynamin at a single site that defines a new SH3 binding consensus sequence. J Biol Chem. 1997; 272(20): 13419-25.

15. Yoshida Y, Takei K. Stimulation of dynamin GTPase activity by amphiphysin. Methods Enzymol. 2005; 404: 528-37.

16. Dawson JC, Legg JA, Machesky LM. Bar domain proteins: a role in tubulation, scission and actin assembly in clathrin-mediated endocytosis. Trends Cell Biol. 2006; 16(10): 493-8.

17. Ferguson SM, et al. Coordinated actions of actin and BAR proteins upstream of dynamin at endocytic clathrin-coated pits. Dev Cell. 2009; 17(6): 811-22.

18. Takei $\mathrm{K}$, et al. Functional partnership between amphiphysin and dynamin in clathrin-mediated endocytosis. Nat Cell Biol. 1999; 1(1): 33-9.

19. Deciphering Developmental Disorders, S., Large-scale discovery of novel genetic causes of developmental disorders. Nature. 2015; 519(7542): 223-8. 